\title{
RESULTS FROM THE DEVELOPMENT AND TESTING OF THE WORLD'S FIRST PRINCIPLES-BASED FIDELITY INDEX FOR

PEER SUPPORT IN MENTAL HEALTH SERVICES

Goldsmith LP1 ${ }^{1}$, Stepanian $\mathrm{K}^{2}$ White $\mathrm{S}^{1}$, Banach $\mathrm{N}^{1}$, Barlow E ${ }^{3}$, Marks J1, McWilliam $\mathrm{C}^{1}$, Morshead $\mathrm{R}^{1}$, Verey $\mathrm{A}^{4}$, Gillard $\mathrm{S}^{1}$

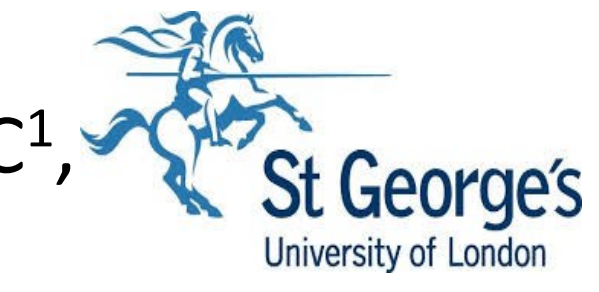

${ }^{1}$ St George's, University of London, Population Health Research Institute, London, U.K. | 2University of Huddersfield, School of Human \& Health Sciences, Huddersfield, U.K | ${ }^{3}$ Sussex Partnership NHS Foundation Trust, Hove, UK | ${ }^{4}$ City, University of London | School of Health Sciences, London, U.K

Background: Research suggests that the distinctiveness of peer support compared to other forms of mental health support can be compromised in large healthcare organisations for a number of reasons, including the difficulty of establishing whether the support given was truly peer support. There is good evidence that the distinctiveness of peer support can be articulated as a set of principles relating to implementation of peer worker roles.

\section{Methods:}

Five principles underpinning one-to-one peer support in mental health services were identified through a literature review and consultation with an expert panel (Gillard et al., 2017). A fidelity index mapping onto these five principles was developed, with supporting semi-structured interviews for use with peer workers, peer worker team leaders and supported peers. Guidelines for conducting interviews, and for scoring the index were developed. These were piloted at independent peer support organisations in U.K. National Health Service peer support services and at the seven sites of the ENRICH trial of peer support for psychiatric discharge. Blind double-rating was used to verify inter-rater reliability and the psychometric properties of the scale send were verified. Experts with lived experience were involved throughout the research (coproduction); (Gillard et al.,

\section{Peer Support Fidelity Index Principles (Gillard et al., 2017).}

1. Safe, trusting relationships based on shared lived experience

2. Mutuality and reciprocity

. The the validation and application of experiential knowledge control strengths, and to build and strengthen connections 2012).

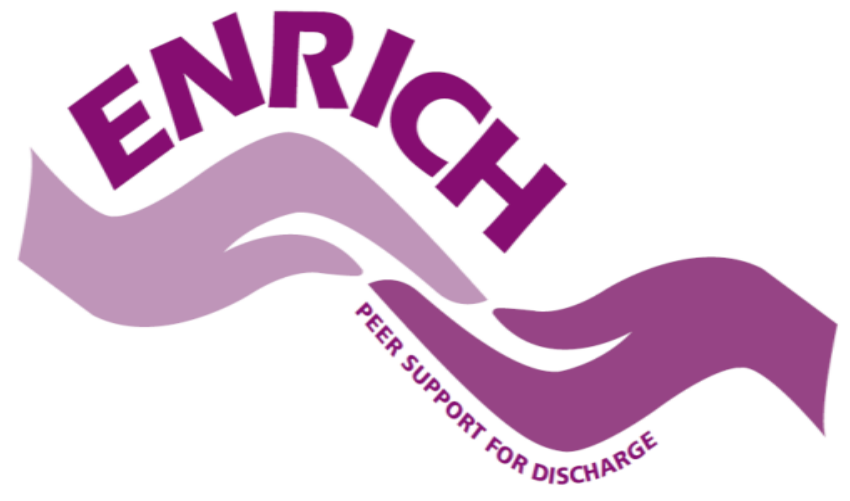

Results: Across the entire index, the total score had an acceptable internal consistency, Cronbach's alpha $=0.81$. The intra-class correlation coefficients were all 0.67 or above, indicating good inter-rater reliability.

Conclusions: The fidelity of peer support in mental health services can be measured in terms of principles that articulate the distinctiveness of peer support. It is acceptable and feasible to develop robust measures of values-based fidelity for complex healthcare interventions where psycho-social and relational mechanisms are core to change processes. Values-based fidelity measures might usefully complement conventional, dosage approaches to trial fidelity. Beyond research purposes, the principlesbased fidelity index can support the implementation and evaluation of peer worker roles. 